\title{
RESEARCH ARTICLE \\ Positioning accuracy assessment of minimally invasive percutaneous injection techniques for the treatment of temporomandibular disorders
}

\author{
${ }^{1}$ Antônio Luís Neto Custódio, ${ }^{2}$ Andrew Cameron, ${ }^{3}$ Mahmoud Bakr, ${ }^{4}$ Chris Little, \\ ${ }^{5}$ Bruno Ramos Chrcanovic and ${ }^{6}$ Peter Reher \\ ${ }^{1}$ Department of Morphology, Institute of Biological Sciences, Federal University of Minas Gerais, Belo Horizonte, MG, Brazil; \\ ${ }^{2}$ School of Dentistry and Oral Health, Griffith University, Gold Coast, Australia; ${ }^{3}$ School of Dentistry and Oral Health, Griffith \\ University, Gold Coast, Australia; ${ }^{4}$ Queensland College of Art, Griffith University, Gold Coast, Australia; ${ }^{5}$ Department of \\ Prosthodontics, Faculty of Odontology, Malmö University, Malmö, Sweden; ${ }^{6}$ School of Dentistry and Oral Health, Griffith \\ University, Gold Coast, Australia
}

Objective: The aim of the present study was to evaluate the accuracy of an extraoral CBCTplanned 3D-printed surgical guide aimed to percutaneous injection of substances into the temporomandibular joint (TMJ) and the lateral pterygoid muscle (LPM).

Methods: Nine human cadaver heads were used. Pre-planning CBCT and facial scans were obtained and three percutaneous injection sites were planned: one for the lower compartment of the TMJ and two for the LPM. A digital surgical guide was then designed with small titanium sleeves and printed by a 3D printer. After the injections, new CBCT scans with the needles in place were obtained in order to assess the accuracy of the procedure in relation to the virtual planning.

Results: The mean values for angle deviation were very low (range $1.13^{\circ}-4.08^{\circ}$ ), the same happening for the mean difference in the length reached (range $1.82-2.64 \mathrm{~mm}$ ), as well as for the mean difference in the needle tip dislocation (range $0.94-2.03 \mathrm{~mm}$ ).

Conclusion: The guide seems to be a reliable tool for accurate percutaneous injection of drugs into the inferior compartment of the TMJ and the LPM. Further studies are necessary to test the efficacy and validate the method in an in vivo study.

Dentomaxillofacial Radiology (2020) 49, 20200313. doi: 10.1259/dmfr.20200313

Cite this article as: Custódio ALN, Cameron A, Bakr M, Little C, Chrcanovic BR, Reher P. Positioning accuracy assessment of minimally invasive percutaneous injection techniques for the treatment of temporomandibular disorders. Dentomaxillofac Radiol 2020; 49: 20200313.

Keywords: Temporomandibular joint; lateral pterygoid muscle; 3D printing; surgical guide; percutaneous injection

\section{Introduction}

The temporomandibular joints (TMJ) are the bilateral connections between the temporal bones of the skull and the mandible. As with any other joint in the human body, the TMJ is also subjected to articular disorders, which affect the jaw muscles, the joints and/or the fibrous tissue connecting them. Several therapies have

Correspondence to: Bruno Ramos Chrcanovic, E-mail: bruno.chrcanovic@ mau.se; brunochrcanovic@hotmail.com

Received 03 July 2020; revised 15 July 2020; accepted 15 July 2020 been proposed to change the intracapsular environment and relieve TMJ symptoms. ${ }^{1}$ These therapies usually require injections of substances into the superior or inferior articular compartments of the TMJ.

Several studies have used freehand manual needle insertion in order to inject substances into the TMJ space, usually guided by electromyography. ${ }^{1-4} \mathrm{~A}$ more invasive approach is the injection guided by arthroscopy. ${ }^{5}$ Any TMJ puncture has, however, the potential 
risk of complications such as arterial bleeding, preauricular and extradural haematoma or even intracranial perforation. ${ }^{6,7}$ Therefore, it is of the utmost importance to plan very well any procedure designed to inject substances into the TMJ.

CT-derived stereolithographic surgical guides can be used to provide a more precise implant placement and to reduce the risk of damage to adjacent structures. ${ }^{8,9}$ The surgical guide is fixed in place in the oral cavity, making high-precision implant placement possible. These guides can be modified for other applications such as the precise insertion of needles. The aim of this study was to evaluate the accuracy of an extraoral CBCT-planned 3D-printed surgical guide aimed to percutaneous injection of substances into the TMJ and the lateral pterygoid muscle (LPM).

\section{Methods and materials}

\section{Aims}

The purpose of the present study was to evaluate the accuracy of an extraoral CBCT-planned 3D-printed surgical guide aimed to percutaneous injection of substances into three different locations of the TMJ spaces, namely the anterior and posterior regions of the LPM, and the lower compartment of the TMJ.

\section{Materials}

For the development of this project, nine human cadaver heads (mean age $\pm \mathrm{SD}, 89.9 \pm 6.3$ years) were used, from the School of Medical Science, Griffith University, Queensland, Australia. The Griffith University Human Research Ethics Committee determined that the project did not require ethical review and approval, based on the assurance that the proposed research only involved experimentation on the heads of cadavers (see acknowledgements).

\section{Selection criteria}

The selection criteria consisted of specimens with a minimum jaw opening of $3 \mathrm{~cm}$.

\section{$3 D$ surgical guide planning and manufacture}

Pre-planning CBCT scan in axial and coronal planes was obtained with a Carestream 9300 (Carestream Health Inc., Atlanta, GA, USA). The scans were acquired using the Dental Imaging Software v.6.14.7.3 (Carestream Health Inc., Atlanta, USA) and included the TMJ region on both sides of each cadaver. The following CBCT scan parameters were used for all specimens: a tube voltage of $85 \mathrm{kV}, 4.0 \mathrm{~mA}$, emission of X-rays over an interval of $8.0 \mathrm{~s}$ and an effective dose of $389 \mathrm{mGy} / \mathrm{cm}^{2}$. The resolution was set to 180 voxels $/ \mathrm{mm}^{3}$, allowing for $0.1-\mathrm{mm}$-thick scans and the field of view was $17.0 \times 13.5 \mathrm{~cm}$.

Facial scans of the specimens were performed with a smart phone (iPhone 11, Apple Inc., Cupertino, CA, USA) on a facial scanning application (Bellus 3D Dental

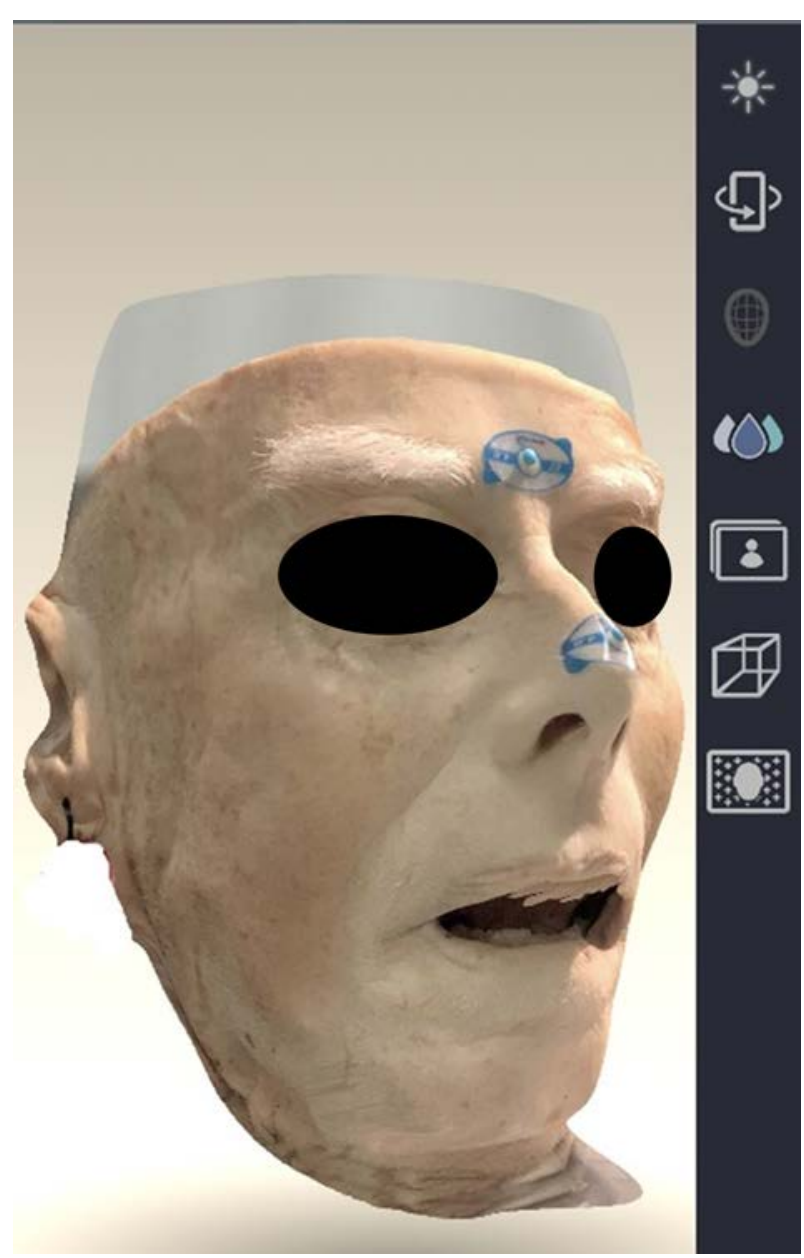

Figure 1 Scan of cadaver head obtained with Bellus 3D Dental Pro.

Pro, Bellus 3D Inc., Campbell, CA, USA), as seen in Figure 1 . The heads were stabilized using a plastic stand on a turntable and the face was scanned with the following boundaries: the hair implant line (upper boundary), the laryngeal prominence (lower boundary) and the ears (lateral boundary). This application allows the capture of a subject's face and simple export of high-resolution files in a Standard Tessellation Language (STL) format which is a $3 \mathrm{D}$ representation of a given object.

The digital planning phase was performed using an implant planning software (Implant Studio, 3Shape A/S, Copenhagen, Denmark). The STL files of the facial scan were then imported along with the CBCT data. The two data sets were then aligned with the custom alignment tool built into the software (Figure 2). A generic implant fixation pin was then selected to simulate the injection path and site. Three sites have been selected, one for the lower compartment of the TMJ, and two for the LPM. The TMJ injection site was directed towards the posterior and medial aspect of the condyle, aiming for the lower compartment area. The entry point of the needle was then positioned at a distal angulation, so that the sleeve exit point was located close to the tragus. 


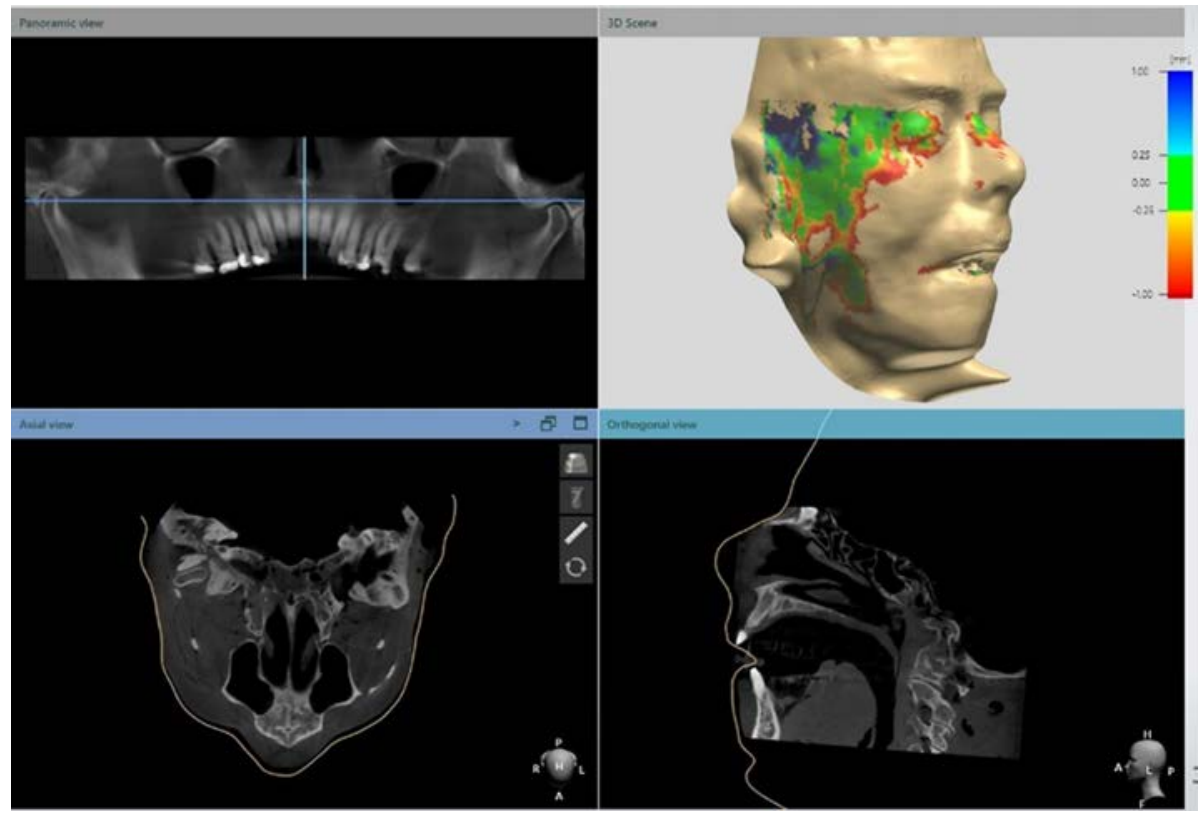

Figure 2 Alignment of the facial scan with the CBCT using the custom alignment tool in Implant Studio 3Shape A/S.

Simulated needles were placed to reach the centre of the LPM. The path of the posterior injection was planned to commence on the skin $0.5 \mathrm{~cm}$ in front of the condyle of the mandible, passing below the zygomatic arch, through the mandibular notch (between coronoid and condylar processes), aiming to reach the LPM more posteriorly. The anterior LPM point was standardized $1.0 \mathrm{~cm}$ anterior to the posterior point, aiming to reach the LPM more anteriorly, following a parallel path as the posterior injection. The implant alignment tool was used to ensure the LPM injections were parallel.

The exact depth of injection for each TMJ was calculated from pre-planning CBCT exams. The needles were planned to be inserted at a $90^{\circ}$ angle in relation to the skin, with the mandible at closed mouth position. The pre-planning CBCT scans were analysed to check for any abnormalities within the TMJ's compartments that might affect the technique. Depth measurements for the

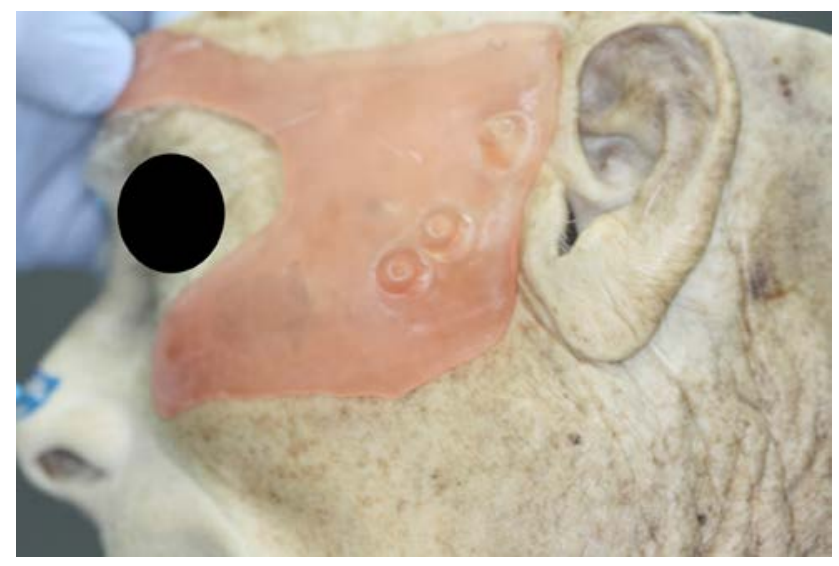

Figure 3 Surgical guide testing of adaption to cadaver head. insertion of the needles into the TMJ and LPM were taken during surgical planning and exported in the surgical plan.

A digital surgical guide was then designed with small titanium sleeves (Steco-System Technik GmbH \& Co. $\mathrm{KG}$, Hamburg, Germany) with an internal diameter of $1.16 \mathrm{~mm}$ and length of $5 \mathrm{~mm}$. The sleeves were positioned juxtaposed from the surface of the cadavers' skin. A surgical guide was then designed on the STL mesh of the facial scan. It was designed to have extensions covering key anatomical landmarks including supraorbital and infraorbital rims, zygomatic prominence and the mandible and TMJ area, providing stability of the guide over the skin.

The final digital design of the surgical guide was then exported as an STL file. This file was imported into the PreForm 3D Printing Software (FormLabs, Somerville, MA, USA) in order to nest and slice in preparation for $3 \mathrm{D}$ printing. This file was then imported to a Form 2 Stereolithography 3D Printer (FormLabs, Somerville, MA, USA), and the guide was printed in FormLabs Surgical Guide Resin (FormLabs, Somerville, MA, USA) with a layer thickness set to $50 \mu \mathrm{m}$. Once the printing was completed, post processes were followed as per manufacturers' instructions, the support structures were removed, the guide was then assessed for fit prior to the titanium sleeves being inserted into their positions as planned (Figure 3). A final cure in Form Cure (FormLabs, Somerville, MA, USA) was then performed.

\section{Accuracy evaluation of injections}

The selected heads were removed from the refrigerator $12 \mathrm{~h}$ before the experiment, to facilitate manipulation of the jaw. The right and left guides were stabilized in the 


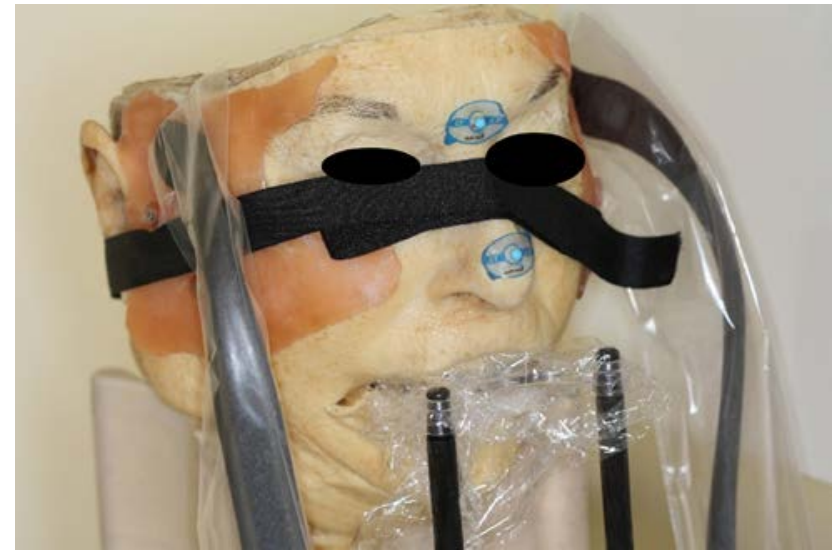

Figure 4 Cadaver in $\mathrm{CBCT}$ machine with guides in place prior to needle insertion and scanning.

correct position with the aid of velcro tape as seen in Figure 4.

For the LPM, two similar needles (BD Spinal Needle, Ref. 405249, 19 GA, $1.1 \times 75 \mathrm{~mm}$; Becton Dickinson, Franklin Lakes, NJ, USA) were used. For the inferior compartment of the TMJ, a shorter needle was used (BD PrecisionGlide TM Needle, Ref 301828, 19 G 1 1⁄2 TW, $1.1 \times 38 \mathrm{~mm}$; Becton Dickinson, Franklin Lakes, NJ, USA). The needles depth of insertion was marked with a silicone cursor as per planning (Figure 5).

New CBCT scans with the needles in place were performed to evaluate the actual positioning of each needle in relation to what was planned.

\section{Data collection}

The CBCT scans (pre-planning and after insertion of the needles) were imported into Intellispace Portal 9.0 (Phillips, Amsterdam, Netherlands) and a 3D solid mesh of the scans was created for extrapolation of the needle path of insertion (Figure 6). The following measurements were obtained: (1) the angle of deviation between the planned versus actual insertion path of the needle and (2) the distance from the planned target location of

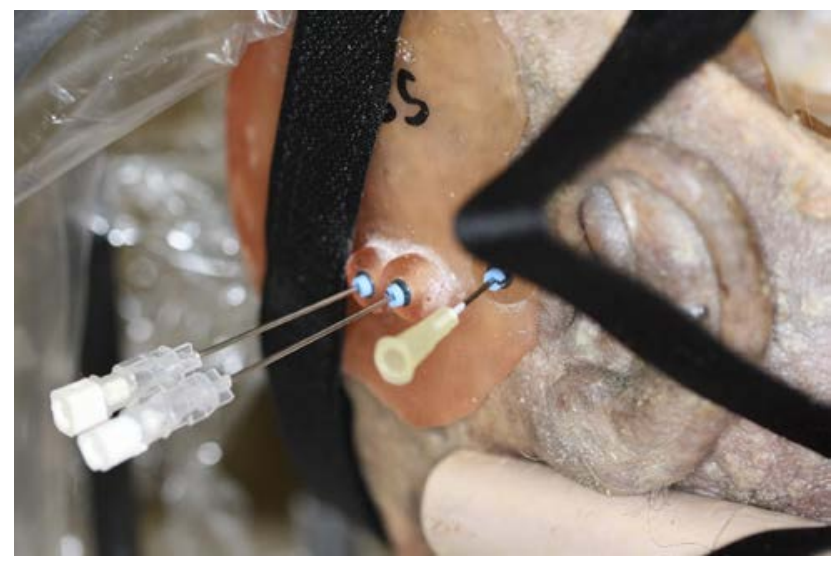

Figure 5 Needles in situ showing premeasured depth stoppers for needles.

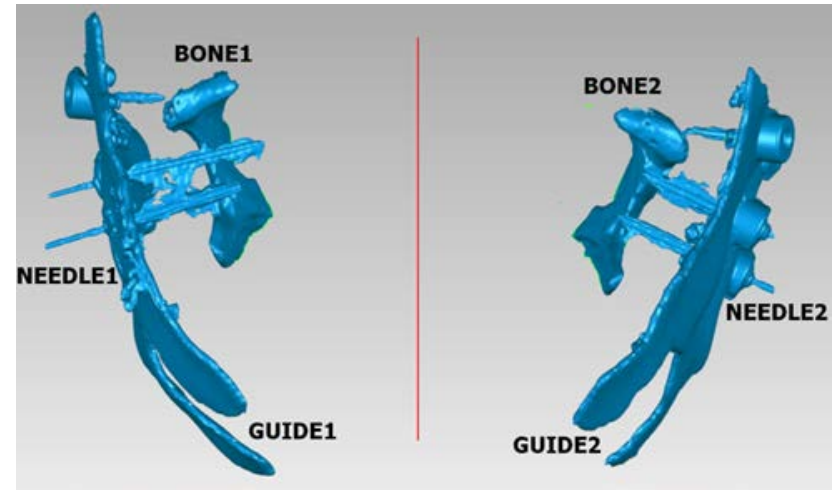

Figure 6 Extraction of needle positioned in relation to original planned guide position using Intellispace Portal software.

the needle tip to the actual position after the insertion, in two ways: (a) the difference in the length reached and (b) the difference in the tip dislocation (other than the length). The angle and the distances were measured utilizing Rhinoceros 3D ${ }^{\circledR}$ (Robert McNeel\& Assoc., Boston, USA), as seen in Figure 7. The data were then imported into a SPSS v.26 (IBM Corp., Armonk, NY, USA) file.

\section{Statistical analyses}

A descriptive analysis was performed based on mean, standard deviation (SD), median, range and percentage values. All data were analysed using IBM SPSS Statistics for Windows, v.26.0 (IBM Corp., Armonk, NY, USA).

\section{Results}

Nine human cadaver heads were used for the present study, which would provide 18 TMJs for analysis. However, for one TMJ, the needles were scattered severely in the CBCT scan to a point that we could only speculate that the needle or the specimen became unsteady and for some reason was moved sideways during scanning. Therefore, this one TMJ was excluded from the present results.
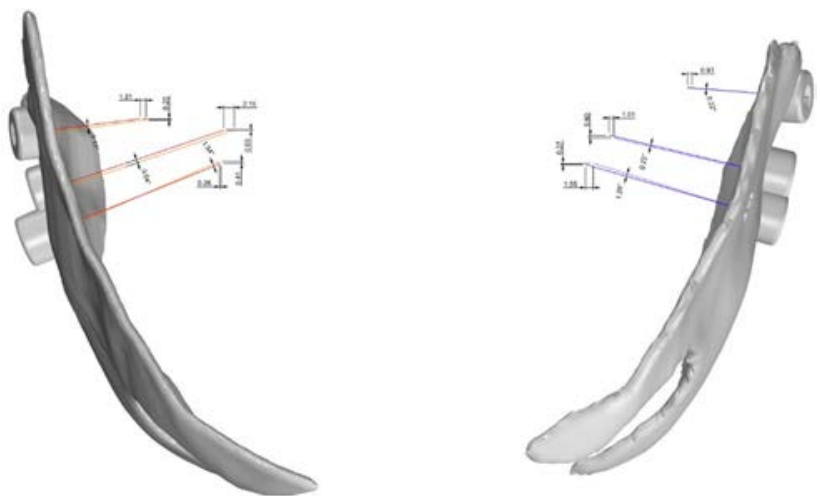

Figure 7 Deviation of planned $v s$ actual position of needles measurements using Rhinoceros $3 \mathrm{D}$ software. 
Table 1 Angle of deviation between the planned $v s$ actual insertion path of the needle, and the distance from the planned target location of the needle tip to the actual position after the insertion, according to the injection location and the CT plane

\begin{tabular}{|c|c|c|c|c|c|}
\hline Plane & & $n$ & $\begin{array}{c}\text { Angle (degrees) } \\
\text { Mean } \pm S D(\text { median })(\min , \max )\end{array}$ & $\begin{array}{c}\text { Length }(\mathrm{mm}) \\
\text { Mean } \pm S D(\text { median })(\text { min, max })\end{array}$ & $\begin{array}{c}\text { Dislocation }(\mathrm{mm}) \\
\text { Mean } \pm S D(\text { median })(\min , \max )\end{array}$ \\
\hline \multirow{3}{*}{ Axial } & LPM-anterior & 17 & $2.55 \pm 1.96(2.19)(0.18,7.88)$ & $1.82 \pm 2.15(1.05)(0.26,8.28)$ & $1.53 \pm 1.17(1.33)(0.26,3.77)$ \\
\hline & LPM-posterior & 17 & $1.13 \pm 0.98(0.87)(0.04,2.59)$ & $1.97 \pm 1.96(1.23)(0.11,7.94)$ & $0.94 \pm 0.78(0.70)(0.03,2.68)$ \\
\hline & Inferior TMJ & 17 & $2.30 \pm 2.31(2.07)(0.17,8.87)$ & $2.64 \pm 1.92(2.12)(0.08,6.21)$ & $1.04 \pm 0.96(0.87)(0.00,3.12)$ \\
\hline \multirow{3}{*}{ Coronal } & LPM-anterior & 17 & $2.91 \pm 2.39(2.67)(0.22,9.22)$ & $1.85 \pm 2.15(1.05)(0.26,8,28)$ & $2.03 \pm 1.71(1.65)(0.07,6.69)$ \\
\hline & LPM-posterior & 17 & $2.77 \pm 1.91(2.34)(0.16,6.97)$ & $1.94 \pm 1.97(1.22)(0.11,7.94)$ & $1.70 \pm 1.47(1.41)(0.01,5.48)$ \\
\hline & Inferior TMJ & 17 & $4.08 \pm 2.25(4.59)(0.37,8.07)$ & $2.64 \pm 1.92(2.12)(0.08,6.21)$ & $1.92 \pm 1.40(1.42)(0.21,4.85)$ \\
\hline
\end{tabular}

SD-standard deviation, min-minimum, max-maximum

LPM-anterior-anterior region of the lateral pterygoid muscle

LPM-posterior-posterior region of the lateral pterygoid muscle

InferiorTMJ-inferior compartment of temporomandibular joint

TMJ Table 1 shows the angle of deviation between the planned vs actual insertion path of the needle, and the distance from the planned target location of the needle tip to the actual position after the insertion, according to the injection location and the CT plane. The mean values for angle deviation were very low, ranging from $1.13^{\circ}$ to $4.08^{\circ}$, the same happening for the mean difference in the length reached (range 1.82 to $2.64 \mathrm{~mm}$ ), as well as for the mean difference in the needle tip dislocation (range 0.94 to $2.03 \mathrm{~mm}$ ). All except one of the measurements presented median values lower than the mean values.

\section{Discussion}

The percutaneous injection approaches evaluated here were described by other studies, ${ }^{1,4}$ but without the use of a surgical guide. To the best of the authors' knowledge, the present work presents the second study on extraoral CBCT-planned 3D-printed surgical guide aimed for use in TMJ therapy. The first one was described by Krause et al, ${ }^{10}$ planned for endoscopic surgery of the TMJ. Other studies also made use of surgical guides for the injection of drugs, but using intraoral instead of extraoral guides. ${ }^{11-13}$ The present approach allows planning of the exact position of the injection target area, which might make infiltration of substances to the TMJ easier and safer for novices in the field. The depth of the puncture is regulated by marking the puncture needle in advance, and the guide assists the operator by allowing easy puncturing at the correct location.

The approach proposed in this study can be used for the injection of botulinum toxin aimed for the treatment of severe TMJ clicking, ${ }^{2}$ TMJ dislocation ${ }^{4}$ or LPM dystonia. ${ }^{5}$ When it comes to the inferior TMJ compartment, the technique can be used for the application of hyaluronic acid in viscosupplementation ${ }^{14}$ or access for arthrocentesis. ${ }^{10}$ Patients who will not benefit from this approach consist of the same that would not be indicated to be submitted to the aforementioned procedures.

The present technique showed promising results. The mean values of the angle of deviation between the planned $v s$ actual insertion path of the needle and of the distance from the planned target location of the needle tip to the actual position after the insertion were very low. The cases that presented a greater deviation from the planned needle insertion may be either result of the difficulty to properly stabilize the guide on the skin of cadavers or the small variations in the position of the heads in the CBCT machine between the pre- and post-procedure radiological exams.

It is difficult to compare the present results with the ones from other studies, since only one other study evaluated on the use of an extraoral CBCT-planned 3D-printed surgical guide, ${ }^{10}$ and this did not focus on the accuracy of the technique. Three studies evaluated the use of intraoral guide for the same purpose. ${ }^{11-13}$ One publication reported the use of the technique in only one subject, ${ }^{11}$ and the other two studies ${ }^{12,13}$ did not focus on the accuracy of the procedure.

The present approach has some advantages when compared to the intraoral technique. In patients with an extremely narrow space between the coronoid process and maxilla, it is very difficult to accurately and safely inject substances using the intraoral approach. ${ }^{12}$ In addition, the intraoral approach can provide injection to a limited part of the muscle. ${ }^{13}$ The present percutaneous technique avoids the need of evaluation of electromyographic burst during mouth opening or protrusion in order to correctly place the needle. The technique is also very advantageous when the patient with severe mental impairment, for which injection needs to be done under general anaesthesia ${ }^{13}$ and the correct placement need to be verified by eliciting jaw reflex ${ }^{15}$ or by the use of a nerve stimulator. ${ }^{16}$

Another advantage is the decreased risk of complications. Puncturing the TMJ can cause complications such as facial nerve damage, penetration of the middle cranial fossa, injury to the middle ear, or irreversible changes to the TMJ. ${ }^{17-19}$ There is also the risk of complications such as bleeding, haematoma and swelling, ${ }^{12}$ which are related to the injury of the maxillary artery. The maxillary artery is the largest terminal branch of the external carotid artery that arises at the back of the condylar neck of the mandible, and it runs, either superficial or deep to the LPM, to the pterygopalatine fossa. The greater the 
number of times the needle is inserted, the greater the risk of complications and pain. Thus, the more accurately the needle is inserted, the less likely complications are. ${ }^{13}$ The use of a needle guide allows clinicians to perform the injection procedures with a lower risk of complications. Without a guide, operators would require several years to achieve skill levels that would minimize complications. ${ }^{12}$

The technique is not without disadvantages. The treatment will require that the patient incurs additional costs for the laboratory work to fabricate the surgical guide, with the surgical planning made by someone with adequate skills and knowledge. Moreover, the precision of the whole procedure depends largely on the ability to position accurately the guide, and to maintain it stable in position during the whole procedure, ${ }^{8}$ which is more difficult when teeth are not used as a support to stabilize the guide. Despite probably saving time during the procedure, the planning phases in which CBCT and optical scanning are combined is time-consuming.

The use of cadavers may be seen as disadvantageous by some researchers. However, the closest anatomical model to resemble a live patient is a fresh human cadaver, which makes the perfect ground for training of surgeons to a safe level before embarking on to patients. ${ }^{20}$

The limitations of the present study include small sample size and the sole inclusion of specimen with high age. It was not possible to be absolutely sure that the needle reached the inferior TMJ compartment, as this was not checked radiologically by the use of a radiocontrast agent. Last but not least, as this was a cadaver

\section{REFERENCES}

1. Long X, Chen G, Cheng AHan, Cheng Y, Deng M, Cai H, et al. A randomized controlled trial of superior and inferior temporomandibular joint space injection with hyaluronic acid in treatment of anterior disc displacement without reduction. J Oral Maxillofac Surg 2009; 67: 357-61. doi: https://doi.org/10.1016/j.joms.2008.09. 014

2. Bakke M, Møller E, Werdelin LM, Dalager T, Kitai N, Kreiborg S. Treatment of severe temporomandibular joint clicking with botulinum toxin in the lateral pterygoid muscle in two cases of anterior disc displacement. Oral Surg Oral Med Oral Pathol Oral Radiol Endod 2005; 100: 693-700. doi: https://doi.org/10.1016/j.tripleo. 2004.11.019

3. Emara AS, Faramawey MI, Hassaan MA, Hakam MM. Botulinum toxin injection for management of temporomandibular joint clicking. Int J Oral Maxillofac Surg 2013; 42: 759-64. doi: https:// doi.org/10.1016/j.ijom.2013.02.009

4. Fu K-Y, Chen H-M, Sun Z-P, Zhang Z-K, Ma X-C, KY F, XC M. Long-Term efficacy of botulinum toxin type A for the treatment of habitual dislocation of the temporomandibular joint. $\mathrm{Br} \mathrm{J} \mathrm{Oral}$ Maxillofac Surg 2010; 48: 281-4. doi: https://doi.org/10.1016/j. bjoms.2009.07.014

5. Martos-Díaz P, Rodríguez-Campo F-J, Bances-del Castillo R, Altura-Guillén O, Cho-Lee G-Y, Mancha-dela-Plata M, et al. Lateral pterygoid muscle dystonia. A new technique for treatment with botulinum toxin guided by electromyography and arthroscopy. Med Oral Patol Oral Cir Bucal 2011; 16: e96-9. doi: https://doi.org/10. 4317/medoral.16.e96

6. Hohenberger GM, Schwarz AM, Grechenig P, Grechenig C, Krassnig R, Weiglein AH, et al. Success rate in puncture of the study, it was not possible to verify the occurrence of some complications, as it would be possible in an in vivo study.

\section{Conclusion}

The surgical guide presented in the present study seems to be a reliable tool for accurate percutaneous injection of drugs into the inferior compartment of the TMJ and the LPM. Further studies are necessary to test the efficacy and validate the method in an in vivo study.

\section{Acknowledgment}

The authors would like to thank the technical anatomy staff team at the School of Medical Science (Discipline of Anatomy) from Griffith University, especially Bevan Butcher, Theresa Gaynor and Leslie Edwards for their continuous efforts in facilitating research projects.

\section{Ethical approval}

All cadavers used in the current study were part of the Griffith University Body Donation program. All donors consented for the use of their bodies in teaching and research activities. Approval was granted from the Chair (School of Anatomy) to conduct the study. temporomandibular joint. Clin Anat 2020; 33: 683-8. doi: https:// doi.org/10.1002/ca.23489

7. Tvrdy P, Heinz P, Pink R. Arthrocentesis of the temporomandibular joint: a review. Biomed Pap Med Fac Univ Palacky Olomouc Czech Repub 2015; 159: 031-4. doi: https://doi.org/10.5507/bp.2013.026

8. Chrcanovic BR, Oliveira DR, Custódio AL. Accuracy evaluation of computed tomography-derived stereolithographic surgical guides in Zygomatic implant placement in human cadavers. J Oral Implantol 2010; 36: 345-55. doi: https://doi.org/10.1563/AAID-JOI-D-0900074

9. Valente F, Schiroli G, Sbrenna A. Accuracy of computer-aided oral implant surgery: a clinical and radiographic study. Int J Oral Maxillofac Implants 2009; 24: $234-42$.

10. Krause M, Dörfler HM, Kruber D, Hümpfner-Hierl H, Hierl T. Template-Based temporomandibular joint puncturing and access in minimally invasive TMJ surgery (MITMJS) - a technical note and first clinical results. Head Face Med 2019; 1515: 10. https//doi.org/. doi: https://doi.org/10.1186/s13005-019-0194-8

11. Oliveira AT, Camilo AA, Bahia PRV, Carvalho ACP, DosSantos MF, da Silva JVL, et al. A novel method for intraoral access to the superior head of the human lateral pterygoid muscle. Biomed Res Int 2014; 2014: 1-8. doi: https://doi.org/10.1155/2014/432635

12. Yoshida K. Computer-Aided Design/Computer-Assisted Manufacture-Derived needle guide for injection of botulinum toxin into the lateral Pterygoid muscle in patients with oromandibular dystonia. J Oral Facial Pain Headache 2018; 32: e13-21. doi: https:// doi.org/10.11607/ofph.1955

13. Yoshida K. Botulinum neurotoxin injection for the treatment of recurrent temporomandibular joint dislocation with and without 
neurogenic muscular hyperactivity. Toxins 2018; 10: 174. doi: https:// doi.org/10.3390/toxins10050174

14. Fonseca RMDFB, Januzzi E, Ferreira LA, Grossmann E, Carvalho ACP, de Oliveira PG, et al. Effectiveness of sequential Viscosupplementation in temporomandibular joint internal derangements and symptomatology: a case series. Pain Res Manag ; 2018; 2018: 5392538: 1: 92018. doi: https://doi.org/10.1155/2018/5392538

15. Moore AP, Wood GD. Medical treatment of recurrent temporomandibular joint dislocation using botulinum toxin A. Br Dent $J$ 1997; 183(11-12): 415-7. doi: https://doi.org/10.1038/sj.bdj.4809523

16. Vázquez Bouso O, Forteza González G, Mommsen J, Grau VG, Rodríguez Fernández J, Mateos Micas M. Neurogenic temporomandibular joint dislocation treated with botulinum toxin: report of 4 cases. Oral Surg Oral Med Oral Pathol Oral Radiol Endod 2010; 109: e33-7. doi: https://doi.org/10.1016/j.tripleo.2009.10.046

17. Bjørnland T, Rørvik M, Haanaes HR, Teige J. Degenerative changes in the temporomandibular joint after diagnostic arthroscopy. An experimental study in goats. Int J Oral Maxillofac Surg 1994; 23: 41-5. doi: https://doi.org/10.1016/s09015027(05)80326-1

18. Sugisaki M, Ikai A, Tanabe H. Dangerous angles and depths for middle ear and middle cranial fossa injury during arthroscopy of the temporomandibular joint. J Oral Maxillofac Surg 1995; 53: 803-10. doi: https://doi.org/10.1016/0278-2391(95) 90337-2

19. Westesson PL, Eriksson L, Liedberg J. The risk of damage to facial nerve, superficial temporal vessels, disk, and articular surfaces during arthroscopic examination of the temporomandibular joint. Oral Surg Oral Med Oral Pathol 1986; 62: 124-7. doi: https://doi.org/10.1016/0030-4220(86)90028-9

20. Sharma M, Macafee D, Pranesh N, Horgan AF. Construct validity of fresh frozen human cadaver as a training model in minimal access surgery. $J S L S$ 2012; 16: 345-52. doi: https://doi. org/10.4293/108680812X13462882735818 